\title{
Solitary thyroid metastasis from a high grade serous ovarian epithelial carcinoma
}

\begin{abstract}
A 51-year old woman with a history of high grade serous ovarian carcinoma stage IV, due to hepatic lesions, was treated in our Department with surgery and combined chemotherapy resulting in complete remission. She revealed a solitary thyroid metastasis and mediastinal lymphadenopathy on PET CT scan, during the evaluation of an increasing value of serum CA-125 at the time of her follow-up. The patient underwent right thyroid lobectomy and received combined chemotherapy again. She remains, since then, on complete biochemical and imaging remission.
\end{abstract}

Keywords: solitary thyroid metastases, ovarian carcinoma, serum ca125, pet scanning

\author{
Special Issue - 2018 \\ Michael MVaslamatzis,' Tegos Th K,' \\ Stathopoulos Ch,' Georgios Kyriakopoulos, ${ }^{2}$ \\ Alevizopoulos N,' Vasili E,' Kyriou L, ${ }^{3}$ \\ Exarchos D, ${ }^{4}$ Stylianidis G, ${ }^{5}$ Rontogianni F, \\ Chr Kandaraki, ${ }^{7}$ Antypa $\mathrm{H}^{8}{ }^{8}$ Athanassiadi $\mathrm{K}^{9}$ \\ 'Department of Medical Oncology, Evangelismos General \\ Hospital, Greece \\ ${ }^{2}$ Department of Pathology, Evangelismos General Hospital, \\ Greece \\ ${ }^{3}$ Department of Biochemistry, Evangelismos General Hospital, \\ Greece \\ ${ }^{4}$ Department of Computed Tomography, Evangelismos General \\ Hospital, Greece \\ ${ }^{5} 2^{\text {nd }}$ Department of Surgery, Evangelismos General Hospital, \\ Greece \\ ${ }^{6}$ Department of Nuclear Medicine/PET, Evangelismos General \\ Hospital, Greece \\ 'Department of Cytology, Evangelismos General Hospital, \\ Greece \\ ${ }^{8}$ Ultrasound Department, Evangelismos General Hospital, \\ Greece \\ ${ }^{9}$ Department of Cardiothoracic Surgery, Evangelismos General \\ Hospital, Greece
}

Correspondence: Michael MVaslamatzis, Head of the Oncology Department, Evangelismos General Hospital, Greece, Tel 0030 2I3204|824,Email michaelvaslamatzis@gmail.com

Received: April 30, 2017 | Published: November 15, 2018
Abbreviations: CA-125, cancer antigen 125; PET, positron emission tomography; FNA, fine needle aspiration; CT, computed tomo-graphy; SUV, standardized uptake value; FIGO, international federation of gynecology and obstetrics; CDDP, cisplatinum

\section{Introduction}

Metastases of solid tumors to the thyroid gland are very in frequent in clinical practice. The most common sources of metastases to the thyroid gland derive from the clear cell carcinomas of the kidneys, breast cancers and non small cell carcinomas of the lung. Cases of carcinomas of women's inner genital system metastasizing to the thyroid gland have been described only in autopsies studies. The aim of this paper is to present the interesting clinical features, the treatment and the clinical outcome of asymptomatic thyroid metastases in a woman with a high grade ovarian cancer, five years after the first diagnosis of her malignant disease.

\section{Case presentation}

A 51-year-old female was admitted in September 2012 in the Department of Medical Oncology, Evangelismos General Hospital, Greece, with bilateral high grade serous ovarian carcinoma stage IV, according to FIGO classification. The patient was diagnosed after thorough examination for persistent abdominal pain the last 6 months.
The computed tomography showed bilateral ovarian masses and hepatic lesions, without ascites. The CA 125 serum value was 5265U/ $\mathrm{ml}$. The patient on 15/8/2012 underwent typical surgery with bilateral salpingo-oophorectomy, total hysterectomy and omentectomy.

Following surgery, she received nine cycles (due to ongoing response after 3 and 6 cycles respectively) of Paclitaxel/Carboplatin/ Bevacizumab, from $26 / 9 / 12$ to $1 / 5 / 13$, as $1^{\text {st }}$ line treatment. The clinical, laboratory and imaging reevaluation demonstrated very impressive clinical response with, potentially minimal residual hepatic disease. The CA 125 serum value decreased to $21 \mathrm{U} / \mathrm{ml}$.

Investigational second look laparotomy (15/7/13) with multiple hepatic biopsies was negative for residual disease and the patient was submitted to close follow-up. After 8 months Ca-125 began rising again $(658 \mathrm{U} / \mathrm{ml})$, but, owing to the negative imaging examination, the woman remained on follow-up. On 1/9/14 enlarged mediastinal lymph nodes were demonstrated in the chest CT and the PET-CT imaging. The patient was submitted to thoracoscopic procedure and the biopsies revealed lymph node infiltration from the known ovarian carcinoma.

The patient was treated with six cycles of Paclitaxel/Carboplatin, due to platinum sensitive disease, from $14 / 10 / 14$ to $29 / 1 / 15$. Normalization of Ca $125(18 \mathrm{U} / \mathrm{ml})$ was documented. In a new PET- 
CT scan only one aortic arch lymph node was found (SUV:3.5), whose biopsy, after surgical intervention, was negative for malignant infiltration.

The patient was put into follow up for approximately one year. At first, biochemical relapse with increasing values of CA 125 was illustrated, while CT scans of chest and abdomen remained negative till 7/2016. The tumor marker CA 125 continued to increase (1680 U/ $\mathrm{ml}$ ) in August 2016, so we proceeded to a new PET-CT scan in 9/2016. There was pathological uptake in multiple mediastinal, cervical lymph nodes (SUV:14), a thyroid lesion in the right lobe (SUV: 4) (Figure 1) and a hepatic lesion in segment IV (SUV: 3.5).

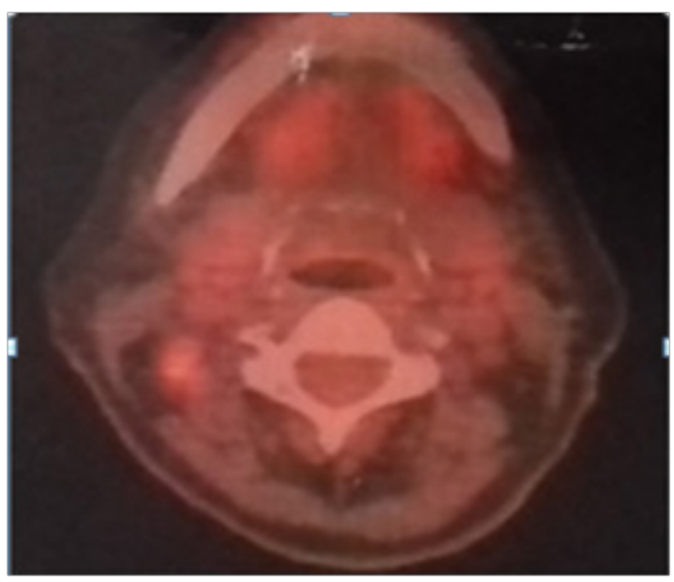

Figure I Thyroid metastasis on PET-CT.

The patient underwent radical node dissection (45 lymph nodes), who's biopsy revealed reactive histiocytosis. FNB of the thyroid lesion (Figure 4) depicted metastatic infiltration from the same ovarian carcinoma. After this diagnosis, a right lobe lobectomy was performed. FNA of the liver, under ultrasound guidance, indicated fatty liver and fibrosis with no signs of malignancy. Thyroid biopsy confirmed the diagnosis of malignant metastatic lesion $2 \times 1,5 \mathrm{~cm}$ by the ovarian carcinoma (Figure $2 \& 3$ ). The 7 local lymph nodes were negative for infiltration from the neoplasm. The patient started chemotherapy with CDDP/Docetaxel with adjuvant intends, after 4 weeks, mainly to treat the potential mediastinal metastatic lymphadenopathy (we have no biopsy from this site at present). The patient is under close follow-up. Following 6 courses of chemotherapy she remains in complete biochemical and imaging remission with an excellent Performance Status (0).

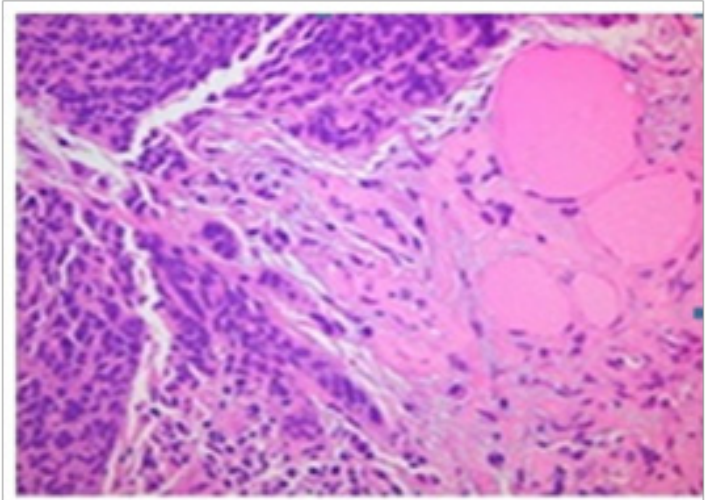

Figure 2 Thyroid infiltration by high grade serous carcinoma with classic cytological figures (H \& E X 200).

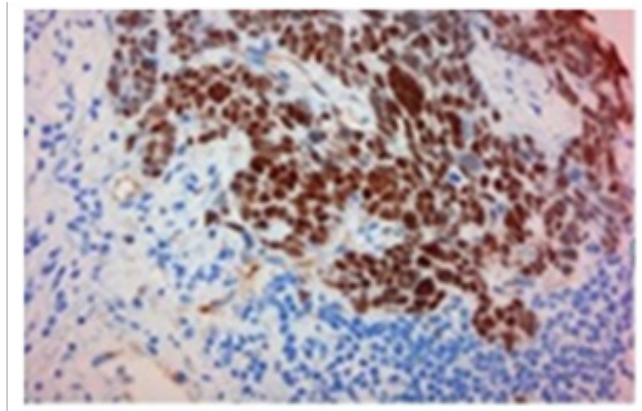

Figure 3 WT-I nuclear expression by the tumor cells (IHC X 200).

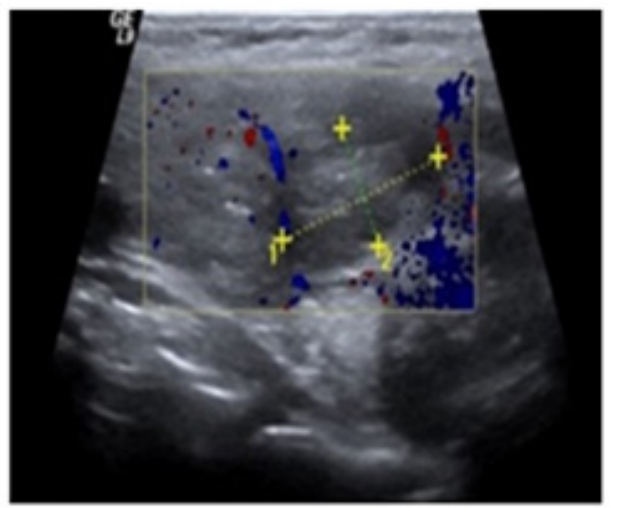

Figure 4 Thyroid metastasis on U/S.

\section{Discussion}

Metastases to the thyroid gland from solid non thyroidal tumors, in autopsy studies, have a frequency between $1.25-24 \%$, but its clinical occurrence, first described in 1960, is very rare. ${ }^{1,2}$ Among 24867 patients treated from $31 / 3 / 1986$ to $31 / 12 / 2016$ in our Department, the aforementioned case is unique $(0.004 \%)$. The age of the woman at diagnosis of thyroid metastases was 51 years, significantly less than the median age (70 years \pm 8.4 , range $46-85$ ) of 35 patients who developed metastases to the thyroid between 2002-2013 in another study. ${ }^{2}$

The commonest primary malignant neoplasms, metastasizing to the thyroid, as reported in clinical series, are kidney carcinomas..$^{3-12}$ Our case (ovarian cancer with thyroid metastasis) is the first one described, at least in the accessible bibliography. Our patient has no symptoms from the metastatic thyroid lesion, as occurs in the majority of the cases, ${ }^{13}$ or a palpable neck mass. Metastases to the thyroid, must not be confused with the primary malignant neoplasm of the gland, or by the direct extension to the thyroid from a locally aggressive tumor of the larynx or the upper esophagus. In these cases the patients often have numerous symptoms such as stridor, dysphagia and dysphonia and palpable neck mass. ${ }^{14}$

The accuracy of thyroid imaging has improved in the era of positron emission tomography (PET scanning), as in our patient, who has been examined unsuccessfully for elevated serum CA 125 levels with conventional imaging techniques. ${ }^{13,15}$ At this point, we would like to note the false positive result of PET in the neck lymph nodes (the histological examination of the radical axillary dissection was negative for malignant infiltration) and liver (FNA of the liver lesion showed fatty liver and fibrosis). The patient underwent right lobectomy, as recommended. ${ }^{7}$ 
Overall prognosis of patients with thyroid metastases appears to be correlated to the type of the primary tumor and the extension of the disease. According to bibliography, $35-80 \%$ of patients with thyroid involvement have synchronous multiorgan metastases. Concerning patients with an indolent primary lesion and disease limited to the thyroid gland without significant extra thyroid extension, outcomes may be favorable. The majority of this group underwent lobectomy or total thyroidectomy and the local long term control seems to be excellent. In contrast, patients with aggressive primary disease, multiorgan metastases, and invasive thyroid metastases will cope less well. ${ }^{13,14}$

\section{Acknowledgements}

None.

\section{Conflict of interest}

The author declares no conflict of interest.

\section{References}

1. Elliott RH Jr, Frantz VK. Metastatic carcinoma masquerading as primary thyroid cancer: a report of authors' 14 cases. Ann Surg. 1960;151:551-561.

2. Cordes M, Kuwert T. Metastases of non-thyroidal tumors to the thyroid gland: a regional survey in middle franconia. Exp Clin Endocrinol Diabetes. 2014;122(5):273-276.

3. Nakhjavani MK, Gharib H, Goellner JR, et al. Metastasis to the thyroid gland. A report of 43 cases. Cancer. 1997;79(3):574-578.

4. Chen H, Nicol TL, Udelsman R. Clinically significant, isolated metastatic disease to the thyroid gland. World J Surg. 1999;23(2):177-180.

5. Wood K, Vini L, Harmer C. Metastases to the thyroid gland: the Royal Marsden experience. Eur J Surg Oncol. 2004;30:583-588.
6. Mirallie E, Rigaud J, Mathonnet M, et al. Management and prognosis of metastases to the thyroid gland. J Am Coll Surg. 2005;200(2):203207.

7. Cichon S, Anielski R, Konturek A, et al. Metastases to the thyroid gland: seventeen cases operated on in a single clinical center. Langenbecks Arch Surg. 2006;391(6):581-587.

8. Russell JO, Yan K, Burkey B, et al. Nonthyroid metastasis to the Thyroid gland: Case Series and Review with Observations by Primary Pathology. Otolaryngol Head Neck Surg. 2016;155(6):961-968.

9. Smith SA, Gharib H, Goellner JR. Fine-needle aspiration. Usefulness for diagnosis and management of metastatic carcinoma to the thyroid. Arch Intern Med. 1987;147(2):311-312.

10. Kim TY, Kim WB, Gong G, et al. Metastasis to the thyroid diagnosed by fine-needle aspiration biopsy. Clin Endocrinol (Oxf). 2005;62(2):236241

11. Dequanter D, Lothaire $P$, Larsimont D, et al. Intrathyroid metastasis: 11 cases. Ann Endocrinol (Paris). 2004;65(3):205-208.

12. Choi SH, Baek JH, Ha EJ, et al. Diagnosis of metastasis to the thyroid gland: comparison of core-needle biopsy and fine-needle aspiration. Otolaryngol Head Neck Surg. 2016;154(4):618-625.

13. Nixon IJ, Coca-Pelaz A, Kaleva AI, et al. Metastasis to the thyroid gland: a critical review. Ann Surg Oncol. 2016;24(6):1533-1539.

14. Chung AY, Tran TB, Brumund KT, et al. Metastases to the thyroid: a review of the literature from the last decade. Thyroid. 2012;22(3):258268.

15. Peparini N, Di Matteo FM, Maturo A, et al. Unusual metastasis of gastrointestinal stromal tumor misdiagnosed as anaplastic thyroid carcinoma. Int J Surg. 2008;6(5):415-417. 